University of New Orleans

ScholarWorks@UNO

2012

\title{
Synthesis and Piezoelectric Response of Cubic and Spherical LiNb03 Nanocrystals
}

\author{
Debasish Mohanty \\ University of New Orleans \\ Girija S. Chaubey \\ University of New Orleans, gchaubey@uno.edu \\ Amin Yourdkhani \\ University of New Orleans, ayourdkh@uno.edu \\ Shiva Adireddy \\ University of New Orleans, spadired@uno.edu \\ Gabriel Caruntu \\ University of New Orleans, gcaruntu@uno.edu
}

See next page for additional authors

Follow this and additional works at: https://scholarworks.uno.edu/chem_facpubs

Part of the Chemistry Commons

\section{Recommended Citation}

RSC Advances, 2012, 2, 1913-1916

This Article is brought to you for free and open access by the Department of Chemistry at ScholarWorks@UNO. It has been accepted for inclusion in Chemistry Faculty Publications by an authorized administrator of ScholarWorks@UNO.For more information, please contact scholarworks@uno.edu. 
Authors

Debasish Mohanty, Girija S. Chaubey, Amin Yourdkhani, Shiva Adireddy, Gabriel Caruntu, and John B. Wiley 


\title{
RSC Advances
}

\section{Synthesis and piezoelectric response of cubic and spherical $\mathrm{LiNbO}_{3}$ nanocrystals $\dagger$}

\author{
Debasish Mohanty, Girija S. Chaubey, Amin Yourdkhani, Shiva Adireddy, Gabriel Caruntu and John B. Wiley*
}

Received 23rd August 2011, Accepted 19th November 2011

DOI: $10.1039 / \mathrm{c} 2 \mathrm{ra00628f}$

\begin{abstract}
Methods have been developed for the shape-selective synthesis of ferroelectric $\mathrm{LiNbO}_{3}$ nanoparticles. Decomposition of the single-source precursor, $\mathrm{LiNb}(\mathrm{O}-\mathrm{Et})_{6}$, in the absence of surfactants, can reproducibly lead to either cube- or sphere-like nanoparticles. X-Ray diffraction shows that the $\mathrm{LiNbO}_{3}$ nanoparticles are rhombohedral $(R 3 c)$. Sample properties were examined by piezoresponse force microscopy (PFM) and Raman where both sets of nanoparticles exhibit ferroelectricity. The longitudinal piezoelectric coefficients, $d_{33}$, varied with shape where the largest value was exhibited in the nanocubes (17 $\mathrm{pm} \mathrm{V}^{-1}$ for the cubes versus $12 \mathrm{pm} \mathrm{V}^{-1}$ for spheres).
\end{abstract}

\section{Introduction}

For the past several decades, there has been considerable interest in the synthesis of ferroelectric materials due to their various applications in electronics, nonvolatile memories, and thin film capacitors. ${ }^{1-2}$ Lithium niobate $\left(\mathrm{LiNbO}_{3}\right.$, here after abbreviated as $\mathrm{LN}$ ) is a well known ferroelectric material and due to its excellent piezoelectric and nonlinear optical properties, has been intensely studied for applications in holographic memories, second harmonic generation, and electrooptics. ${ }^{3-6}$ Further, LN crystals show a strong room temperature spontaneous polarization of $70 \mathrm{C} \mathrm{cm}^{-2}$ that can persist up to the high ferroelectric transition temperature of $1483 \mathrm{~K}$, making it a promising candidate for a number of other applications in sensor and piezoelectric antenna arrays. ${ }^{7-8}$

A variety of synthetic methods have been used to prepare bulk LN including sol-gel, ${ }^{9}$ molten salt synthesis, ${ }^{10}$ hydrothermal methods, ${ }^{11}$ combustion, ${ }^{12}$ and solvothermal methods. ${ }^{13}$ The physical properties of materials can be strongly dependent upon particle shape, size, and crystallinity as well as preparation methods and as such, there is always an interest in studying properties as a function of dimensions, morphology, and processing history. Recently nanoparticles of LN were prepared using soft-chemical, ${ }^{5}$ sugar-PVA matrix, ${ }^{14}$ and solution-phase methods. ${ }^{15}$ One report of the use of a single-source precursor, in combination with a surfactant, has been published and this method produced rod-like structures of aggregated LN nanoparticles. ${ }^{15}$ Despite the use of these various methodologies to prepare nanoscale $\mathrm{LN}$, there have been no reports demonstrating effective shape control in the production of single crystalline nanoparticles. Herein, we describe the controlled synthesis of cube- and sphere-like LN nanoparticles

Department of Chemistry and Advanced Materials Research Institute, University of New Orleans, New Orleans, LA 70148, USA.

E-mail: jwiley@uno.edu; Fax: +1 504280 6860; Tel: +1 5042806849

$\uparrow$ Electronic supplementary information (ESI) available: TEM image,

IR spectra, XRD data. See DOI: 10.1039/c2ra00628f via the single-source precursor, $\mathrm{LiNb}(\mathrm{O}-\mathrm{Et})_{6}$. Both morphologies exhibit ferroelectric response with the cubic crystal demonstrating a larger piezoamplitude.

\section{Experimental section}

LN nanoparticles were readily synthesized by a solvothermal approach. The single-source precursor, $\mathrm{LiNb}(\mathrm{O}-\mathrm{Et})_{6}$ (Alfa Aesar, 5\% w/v in ethanol), and 1,4-butanediol (Sigma-Aldrich, $\geqslant 99 \%$ ), used without any further purification, were heated in an autoclave (Parr Instrument Model \#4749). In typical syntheses, $3.5 \mathrm{ml}$ (for cube-shaped particles) or $4.5 \mathrm{ml}$ (for spherical particles) of $\mathrm{LiNb}(\mathrm{O}-\mathrm{Et})_{6}$ solution were placed in a $\sim 23 \mathrm{ml}$ Teflon sleeve along with $2.5 \mathrm{ml}$ of 1,4-butanediol. The Teflon vessel was sealed in the stainless steel autoclave and heated in a furnace at $235{ }^{\circ} \mathrm{C}$ for $3 \mathrm{~d}$ before being cooled down to room temperature naturally. The resulting white precipitate was collected by centrifugation, washed several times with ethanol, and dried in an oven at $70{ }^{\circ} \mathrm{C}$.

The X-ray diffraction (XRD) patterns of samples were collected on a Philips X-pert PW 3040 MPD X-ray powder diffractometer operated at $40 \mathrm{kV}$ and $40 \mathrm{~mA}$ current with $\mathrm{Cu}-\mathrm{K} \alpha$ radiation. The transmission electron microscopy (TEM) was performed with a JEOL 2010 electron microscope at accelerating voltage of $200 \mathrm{kV}$. Raman spectra were collected at roomtemperature on a Thermo-Fisher DXR dispersive Raman spectrometer in a conventional backward geometry using the $\lambda$ $=532 \mathrm{~nm}$ line with a spectral resolution of $3 \mathrm{~cm}^{-1}$. The laser was focused with an X50 long-focal-length objective to a spot of about $\sim 2 \mu \mathrm{m}$. Measurements were taken with a relatively low power of the incident laser beam $(1 \mathrm{~mW})$, to avoid the overheating of the sample. An Asylum Research MFP-3D atomic force microscope was employed to collect piezoelectric properties in a capacitor geometry, where the conductive AFM tip and the conductive substrate are the top and bottom electrodes, respectively. Individual LN nanoparticles were imaged 
in dual resonance tracking (DART) mode applying a small ac voltage with a drive amplitude of $40 \mathrm{mV}$ and a contact resonance frequency of $270 \mathrm{kHz}$ between the tip and the conductive substrate. To avoid measurement-related artefacts and to compare the piezoelectric properties of LN nanoparticles with different shapes, all measurements were carried out with the same conductive AFM tip and laser spot on the micro-cantilever. Local amplitude and phase hysteresis loops were collected by applying $22 \mathrm{~V}$ ac-voltage between the tip (top electrode) and conductive substrate (bottom electrode).

\section{Results}

Fig. 1 presents the transmission electron microscopy (TEM) images of LN nanoparticles. Both cube-shaped $(\mathrm{a}-\mathrm{d})$ and sphereshaped (e-f) nanoparticles can be obtained by this method. The nanocubes typically have an edge length of 50-60 $\mathrm{nm}$ (based on $\sim 100$ particles, average edge length is $54.32 \pm 6.53 \mathrm{~nm}$ ), while the sphere-like particles have a sphere diameter of $40-60 \mathrm{~nm}$ (ave. dia. $\approx 48.82 \pm 8.53 \mathrm{~nm}$ ). A high resolution TEM image (HRTEM) of a cube, along with its First Fourier Transformation (FFT, see Fig. 1d and its inset), show that it is highly crystalline with the facet oriented along (012) planes. Structural and phase characterization of LN nanoparticles were further performed by X-ray powder diffraction (XRD). From
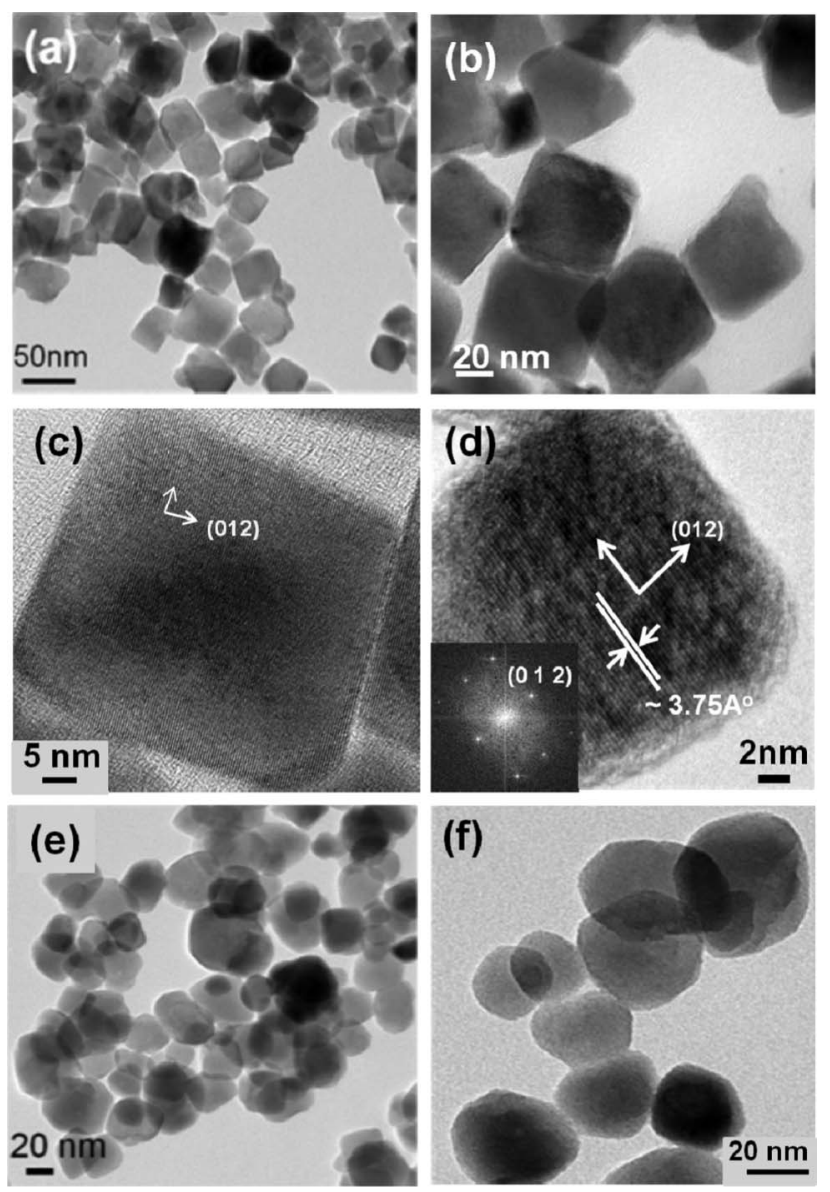

Fig. 1 TEM images of $\mathrm{LiNbO}_{3}$ nanoparticles. (a-d) Cubic shaped particles, (e-f) spherical shaped particles. (d) HRTEM of one single nanocube with its FFT transformation shown as inset.

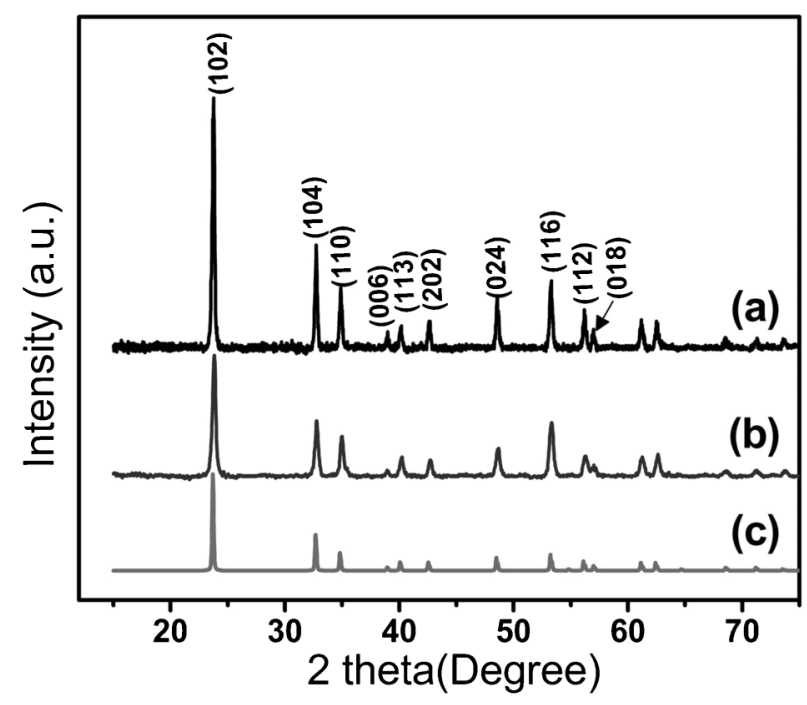

Fig. 2 XRD patterns of $\mathrm{LiNbO}_{3}$ nanoparticles. (a) Nanospheres and (b) nanocubes. The reference pattern (PDF file \#20-0631) of $\mathrm{LiNbO}_{3}$ (trigonal crystal system, space group $R 3 c$ ) is given in (c).

Fig. 2, the nanoparticles can be seen to crystallize in the rhombohedral crystal system of the space group $R 3 c(\# 161)$, this being the ferroelectric phase of LN. All the diffraction peaks are well matched with those from the reference (PDF file \#200631). ${ }^{16}$ The refined lattice parameters are in agreement with the values reported in the literature ${ }^{15}$ and appear to be independent of nanoparticle shape $(a=b=5.145(3) \AA, c=13.867(3) \AA$ for nanocubes; $a=b=5.139$ (3) $\AA, c=13.855$ (3) $\AA$ for nanospheres). Raman spectra in the range of $100-1000 \mathrm{~cm}^{-1}$ are shown in Fig. 3. Peaks are observed at 156, 239, 370, and $433 \mathrm{~cm}^{-1}$ and are attributed to the E transverse optical (TO) phonon mode of LN. These peaks are also consistent with the rhombohedral ferroelectric phase. ${ }^{17,18}$ The fundamental $\mathrm{A}_{1}$ TO modes were observed at 277 and $619 \mathrm{~cm}^{-1}$, which also agree with values reported for ferroelectric LN single crystals. ${ }^{17,18}$

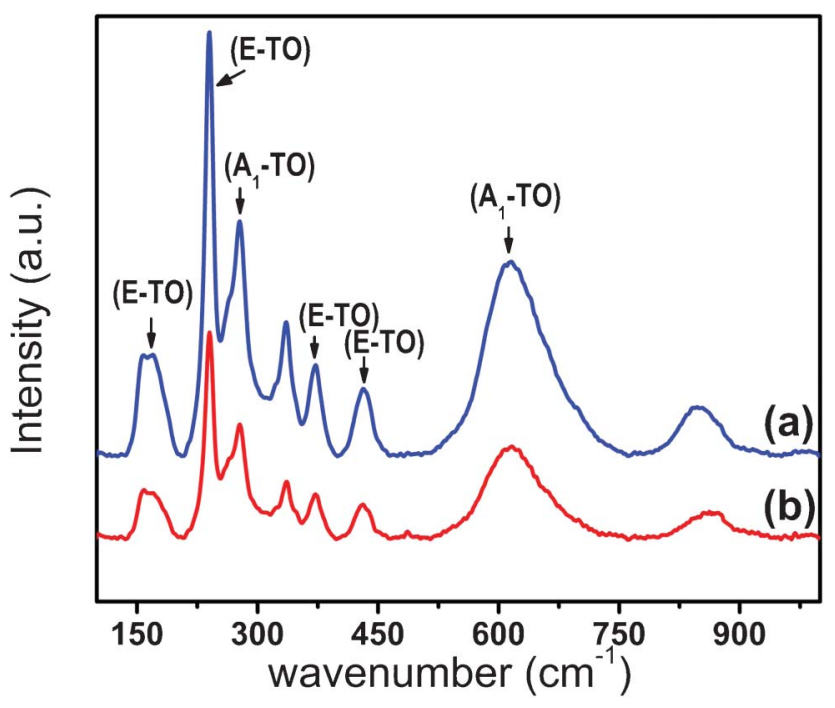

Fig. 3 Raman spectra of $\mathrm{LiNbO}_{3}$ nanoparticles. (a) Nanospheres and (b) nanocubes. Transverse optical (TO) bands assignments, E TO and $\mathrm{A}_{1}$ TO, are shown. 

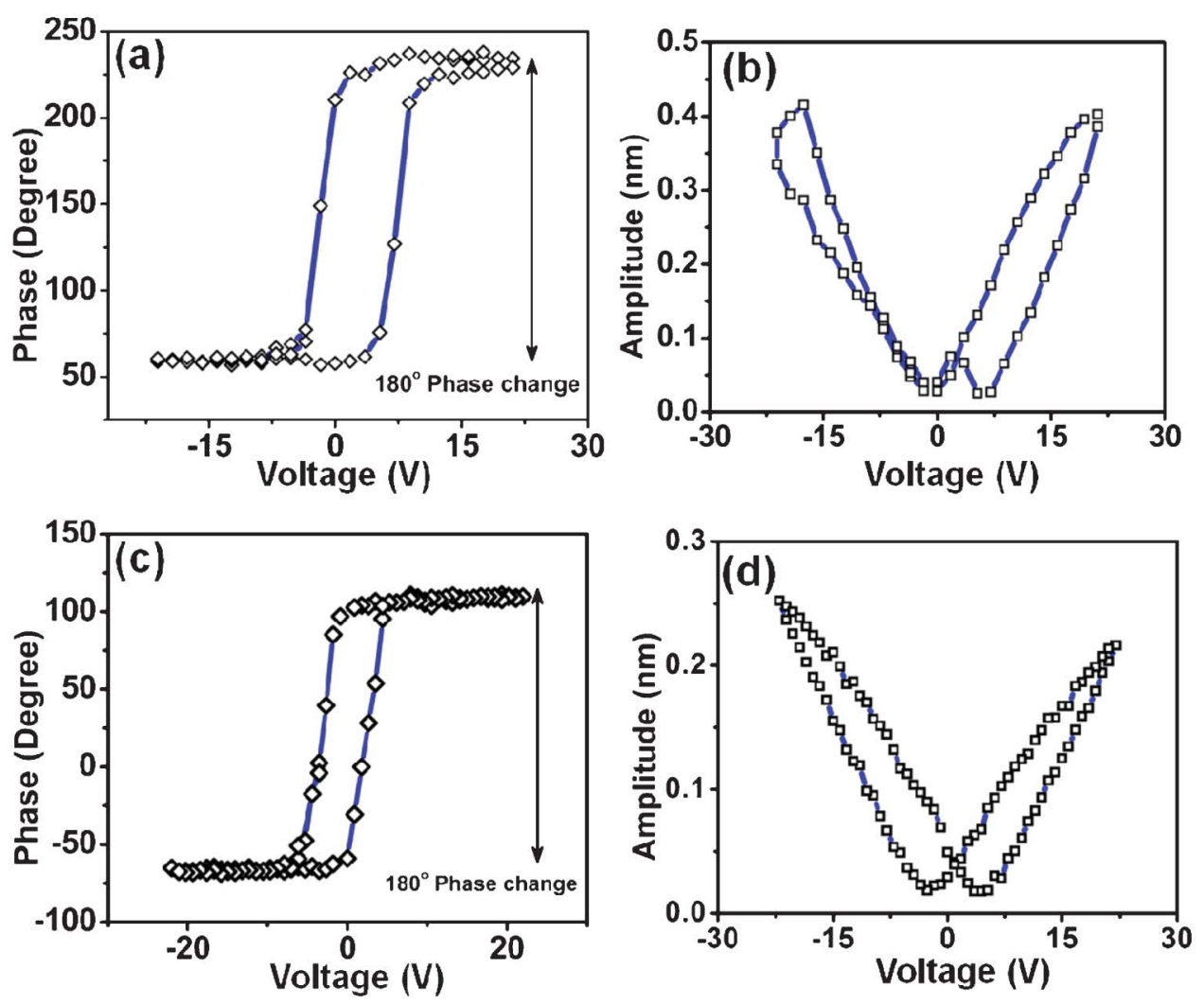

Fig. 4 (a) Phase and (b) amplitude responses from LN nanocubes, (c) phase and (d) amplitude responses from LN nanospheres. The double headed arrows in (a) and (c) highlight the $180^{\circ}$ phase change with voltage.

Piezoresponse force microscopy (PFM) was used to investigate the local electromechanical deformation of LN nanoparticles. Standard PFM hysteresis (piezo-phase versus voltage, Fig. 4a and 4c), and butterfly curves (piezo-amplitude versus voltage, Fig. 4b and $4 \mathrm{~d}$ ), for both $\mathrm{LN}$ nanocubes and nanospheres are shown in Fig. 4. In the phase response curves (Fig. $4 \mathrm{a}$ and $4 \mathrm{c}$ ), the existence of intrinsic lattice polarization in both $\mathrm{LN}$ nanocubes and nanospheres and $180^{\circ}$ switching can be observed, which indicates the existence of $180^{\circ}$ domains in the lattice. ${ }^{19}$ Such a domain change in voltage is generally associated with ferroelectric behavior. ${ }^{20}$ The maximum piezo-amplitude signal measured was approximately $0.4 \mathrm{~nm}$ for the nanocubes and $0.25 \mathrm{~nm}$ for spherical nanoparticles (see Fig. $4 \mathrm{~b}$ and $4 \mathrm{~d}$ ). From the linear portion of the piezoresponse amplitude signal, we have calculated the values of the longitudinal piezoelectric coefficients, $d_{33}$, which are $17 \mathrm{pm} \mathrm{V}^{-1}$ for cube-like and $12 \mathrm{pm} \mathrm{V}^{-1}$ for spherical $\mathrm{LN}$ nanoparticles. $(A=$ $Q V_{\text {ac }} d_{33}$, where $A$ is piezo-amplitude, $Q$ is the quality factor which accounts for the amplitude enhancement at tip-bias resonance and $V_{\text {ac }}$ is the voltage).

\section{Discussion}

The solvothermal method is a versatile technique for the production of metal oxide nanoparticles at low temperatures. In this study we found that the particle shape could be tuned simply by changing the amount of precursor. For larger amounts of $\mathrm{LiNb}(\mathrm{O}-\mathrm{Et})_{6}$, the particles attain nearly spherical shape while with smaller amounts, cubic morphologies with well-defined edges were obtained. In a solvothermal reaction, pressure is generated autogenously in the reaction vessel and is dependent on the total volume of the vessel, the filling factor of the vessel, and the molar volume of the liquids. When the volume of the liquid inside the vessel is increased, the pressure generated decreases according to the Peng-Robinson equation of state. ${ }^{21}$ So by decreasing the amount of precursor, the pressure generated in the reaction vessel increases so as to facilitate the nucleation and growth of the particle along a particular direction, effectively favouring the cubic morphology. 1,4-butanediol also appears to be critical for this synthesis; syntheses attempted with other polyol solvents (ethylene glycol, triethylene glycol) were not successful and we found that only 1,4-butanediol leads to the production of the LN. It was also noted that three days' reaction time was the minimum duration needed to form nanoparticles with definite shape. For example, the TEM image taken from a sample containing nanoparticles obtained after two days of reaction (Fig. $\mathrm{S} 1 \dagger)$ showed only agglomerations of nanoparticles- those particles eventually grow during the aging time to produce nanocubes (Fig. 1a). The reaction temperature $\left(T=235^{\circ} \mathrm{C}\right)$ also has an impact on the stabilization of $\mathrm{LN}$. If the reaction is carried out at $T=250{ }^{\circ} \mathrm{C}$ for three days, the secondary phase, $\mathrm{LiNb}_{3} \mathrm{O}_{8}$, is formed along with LN (Fig. S2 $\dagger$ ) and if the reaction temperature is $220^{\circ} \mathrm{C}$, then crystallization does not occur.

To investigate whether the LN nanoparticles were passivated with solvent species after their growth, FT-IR spectra were collected from neat 1,4-butanediol and then compared to that obtained from a solid sample containing LN nanocubes (Fig. $\mathrm{S} 3 \uparrow$ ). No evidence of 1,4-butanediol can be observed in the 
spectrum. This is also consistent with the surfactant-free approach targeted in this synthesis.

$\mathrm{LN}$ is a ferroelectric material with a high Curie temperature $\left(T_{\mathrm{c}}=1483 \mathrm{~K}\right)$. Below the Curie temperature, the material is rhombohedral with the $R 3 c$ space group, and at higher temperature $\left(>T_{\mathrm{c}}\right)$, changes to $R \overline{3} c$ and $R \overline{3} .{ }^{22}$ In the $R 3 c$ ferroelectric phase, the cations are displaced along the [111] crystallographic direction, which breaks the mirror symmetry plane present in the high temperature paraelectric phase to induce the spontaneous polarization in the lattice. The XRD patterns of the synthesized nanoparticles show that the materials crystallize in the $R 3 c$ phase, and this was further confirmed by Raman phonon bands. The optical phonon modes obtained from Raman scattering are very sensitive to stoichiometry, strain, impurities as well as the crystal system of the materials. ${ }^{17}$ The phonon modes observed for the LN cube-shaped nanoparticles are very similar to those of the spherical nanoparticles both in terms of position and relative intensity (Fig. 3). The characteristic phonon peaks convincingly suggest that the ferroelectric phase $(R 3 c)$ is stable in these nanoparticles, ${ }^{17}$ results which are consistent with the PFM response curves. Close examination of the local piezoelectric response in individual nanoparticles evidenced that the displacement observed in nanocubes is slightly higher than that measured in nanospheres. This difference could be attributed to a greater crystallinity in nanocubes in tandem with the presence of better-defined facets in nanocubes than in spherical nanoparticles. ${ }^{23}$ This is consistent with previous studies where researchers found that piezoelectric properties of nanostructured materials can depend on the crystallite size, ${ }^{23}$ crystal orientation, ${ }^{24}$ and geometry. ${ }^{25,26}$

\section{Conclusions}

Lithium niobate nanoparticles with different morphologies were synthesized under solvothermal conditions by a surfactant-free route using a single-source precursor. The shapes of the nanoparticles, nanocubes versus nanospheres, were tuned by simply changing the concentration of the precursor. Both sets of particles exhibit polarization switching at room temperature with static $d_{33}$ coefficient values of $17 \mathrm{pm} \mathrm{V}^{-1}$ for cube-like and $12 \mathrm{pm} \mathrm{V}^{-1}$ for spherical LN nanoparticles. Work is ongoing to study ferroelectricity in these nanostructures, including the stability of the ferroelectric phase and the kinetics of the local polarization switching in individual nanocrystals. With controlled size and shape and predictable ferroelectric properties, these nanostructures will be attractive as building blocks for the design of complex structures, such as in ferroelectric memories, sensors, and multiferroic nanoparticulate composites.

\section{Acknowledgements}

Financial support from the Department of Defense through DARPA (Grant HR 0011-09-1-0047) is gratefully acknowledged.

\section{References}

1 D. Hennings, M. Klee and R. Waser, Adv. Mater., 1991, 3, 334-340.

2 Z. B. Zhang, X. Z. Sun, M. S. Dresselhaus, J. Y. Ying and J. Heremans, Phys. Rev. B, 2000, 61, 4850-4861.

3 A. Guarino, G. Poberaj, D. Rezzonico, R. Degl'Innocenti and P. Gunter, Nat. Photonics, 2007, 1, 407-410.

4 D. F. Xue and X. K. He, Phys. Rev. B, 2006, 73, 64113.

5 M. Niederberger, N. Pinna, J. Polleux and M. Antonietti, Angew. Chem., Int. Ed., 2004, 43, 2270-2273.

6 D. F. Xue and K. Kitamura, J. Cryst. Growth, 2003, 249, 507-513.

7 I. Inbar and R. E. Cohen, Phys. Rev. B, 1996, 53, 1193-1204.

8 D. L. Zhang, W. J. Zhang, Y. R. Zhuang and E. Y. B. Pun, Cryst. Growth Des., 2007, 7, 1541-1546.

9 M. W. Pitcher, Y. N. He and P. A. Bianconi, Mater. Chem. Phys., 2005, 90, 57-61.

10 P. Afanasiev, Mater. Lett., 1998, 34, 253-256.

11 D. Dey and M. Kakihana, J. Ceram. Soc. Jpn., 2004, 112, 368-372.

12 M. N. Liu, D. F. Xue and C. Luo, J. Am. Ceram. Soc., 2006, 89, $1551-1556$

13 M. N. Liu and D. F. Xue, Mater. Lett., 2005, 59, 2908-2910.

14 S. Wohlrab, M. Weiss, H. C. Du and S. Kaskel, Chem. Mater., 2006, 18, 4227-4230.

15 B. D. Wood, V. Mocanu and B. D. Gates, Adv. Mater., 2008, 20, 4552-4556.

16 National Bureau of Standards (U.S.) Monograph, 1968, 6, 22.

17 A. S. Barker and R. Loudon, Phys. Rev., 1967, 158, 433.

18 R. F. Schaufele and M. J. Weber, Phys. Rev., 1966, 152, 705.

19 S. V. Kalinin, A. N. Morozovska, L. Q. Chen and B. J. Rodriguez, Rep. Prog. Phys., 2010, 73, 056502.

20 S. Jesse, A. P. Baddorf and S. V. Kalinin, Appl. Phys. Lett., 2006, 88, 062908.

21 M. Rajamathi and R. Seshadri, Curr. Opin. Solid State Mater. Sci., 2002, 6, 337-345.

22 C. Abrahams, H. J. Levinstein and J. M. Reddy, J. Phys. Chem. Solids, 1966, 27, 1019.

23 A. Rudiger, T. Schneller, A. Roelofs, S. Tiedke, T. Schmitz and R. Waser, Appl. Phys. A: Mater. Sci. Process., 2005, 80, 1247-1255.

24 Z. X. Cheng, C. V. Kannan, K. Ozawa, H. Kimura and X. L. Wang, Appl. Phys. Lett., 2006, 89, 032901.

25 Z. Y. Wang, J. Hu, A. P. Suryavanshi, K. Yum and M. F. Yu, Nano Lett., 2007, 7, 2966-2969.

26 Y. Zheng, C. H. Woo and B. Wang, J. Phys.: Condens. Matter, 2008, 20, 135216. 